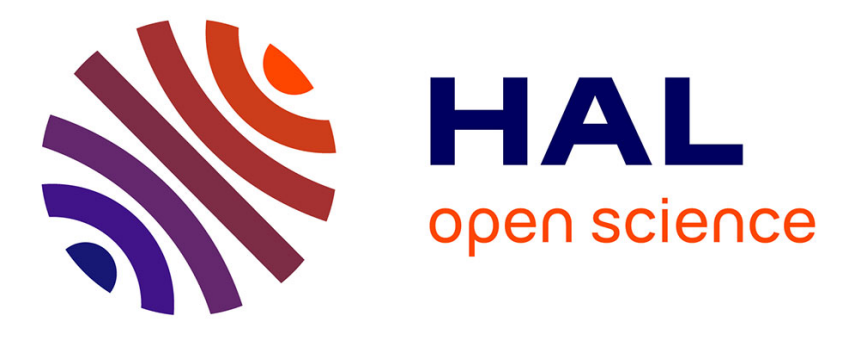

\title{
Between umbra and penumbra
}

\author{
Julien Demouth, Olivier Devillers, Hazel Everett, Marc Glisse, Sylvain \\ Lazard, Raimund Seidel
}

\section{To cite this version:}

Julien Demouth, Olivier Devillers, Hazel Everett, Marc Glisse, Sylvain Lazard, et al.. Between umbra and penumbra. Proceedings of the 23rd Annual Symposium on Computational Geometry, HeeKap Ahn, Otfried Cheong, and Kyung-Yong Chwa, Jun 2007, Gyeongju, South Korea. pp.265-274, 10.1145/1247069.1247117 . inria-00187253

\section{HAL Id: inria-00187253 https://hal.inria.fr/inria-00187253}

Submitted on 14 Nov 2007

HAL is a multi-disciplinary open access archive for the deposit and dissemination of scientific research documents, whether they are published or not. The documents may come from teaching and research institutions in France or abroad, or from public or private research centers.
L'archive ouverte pluridisciplinaire HAL, est destinée au dépôt et à la diffusion de documents scientifiques de niveau recherche, publiés ou non, émanant des établissements d'enseignement et de recherche français ou étrangers, des laboratoires publics ou privés. 
published in Proc. 23th ACM Sympos. on Computational Geometry 2007, p.265-274.

\title{
Between Umbra and Penumbra*
}

\author{
Julien Demouth $^{\dagger} \quad$ Olivier Devillers ${ }^{\ddagger} \quad$ Hazel Everett ${ }^{\dagger}$ \\ Marc Glisse $^{\dagger} \quad$ Sylvain Lazard ${ }^{\dagger} \quad$ Raimund Seidel $^{\S}$
}

\begin{abstract}
Computing shadow boundaries is a difficult problem in the case of non-point light sources. A point is in the umbra if it does not see any part of any light source; it is in full light if it sees entirely all the light sources; otherwise, it is in the penumbra. While the common boundary of the penumbra and the full light is well understood, less is known about the boundary of the umbra. In this paper we prove various bounds on the complexity of the umbra and the penumbra cast by a segment or polygonal light source on a plane in the presence of polygon or polytope obstacles.

In particular, we show that a single segment light source may cast on a plane, in the presence of two triangles, four connected components of umbra and that two fat convex obstacles of total complexity $n$ can engender $\Omega(n)$ connected components of umbra. In a scene consisting of a segment light source and $k$ disjoint polytopes of total complexity $n$, we prove an $\Omega\left(n k^{2}+k^{4}\right)$ lower bound on the maximum number of connected components of the umbra and a $O\left(n k^{3}\right)$ upper bound on its complexity. We also prove that, in the presence of $k$ disjoint polytopes of total complexity $n$, some of which being light sources, the umbra cast on a plane may have $\Omega\left(n^{2} k^{3}+n k^{5}\right)$ connected components and has complexity $O\left(n^{3} k^{3}\right)$.

These are the first bounds on the size of the umbra in terms of both $k$ and $n$. These results prove that the umbra, which is bounded by arcs of conics, is intrinsically much more intricate than the full light/penumbra boundary which is bounded by line segments and whose worst-case complexity is in $\Omega\left(n \alpha(k)+k m+k^{2}\right)$ and $O\left(n \alpha(k)+k m \alpha(k)+k^{2}\right)$, where $m$ is the complexity of the polygonal light source.
\end{abstract}

${ }^{*}$ Research on this paper was initiated at the Fifth McGill-INRIA Workshop on Computational Geometry in Computer Graphics, February 4-10, 2006, held at the Bellairs Research Institute of McGill University in Holetown, St. James, Barbados, West Indies.

${ }^{\dagger}$ LORIA - INRIA Lorraine, \{Firstname.Lastname\}@loria.fr.

‡INRIA Sophia-Antipolis, Olivier.Devillers@inria.fr.

$\S$ Saarland University, FR Informatik, Saarbrücken, Germany, rseidel@cs.uni-sb.de 


\begin{tabular}{|c|c|c|}
\hline Scene type & Lower bounds & Upper bounds \\
\hline \multicolumn{3}{|c|}{ Segment light source } \\
\hline 2 triangles & 4 & $O(1)$ \\
\hline 2 fat polytopes & $\Omega(n)$ & $O(n)$ \\
\hline$k$ polytopes & $\Omega\left(n k^{2}+k^{4}\right)$ & $O\left(n k^{3}\right)$ \\
\hline \multicolumn{3}{|c|}{ n-gon light source } \\
\hline$k$ polytopes & $\Omega\left(n^{2} k^{3}+n k^{5}\right)$ & $O\left(n^{3} k^{3}\right)$ \\
\hline
\end{tabular}

Table 1: Lower bounds on the number of connected components and upper bounds on the complexity of the umbra cast on a plane by $k$ polytopes of total complexity $\mathbf{O}(\mathbf{n})$.

\section{Introduction}

Shadows play a central role in human perception [13, 18]. A wide variety of approaches have been considered for simulating and rendering shadows (see, for example, the surveys $[6,20])$ and many methods make extensive use of graphics hardware (see the survey [10]). Unfortunately, computing realistic shadows efficiently is a difficult problem, particularly in the case of non-point light sources. A part of this difficulty arises from the complicated internal structure that such shadows may have. In this paper we study this structure.

We say that a point is in the umbra if it does not see any part of the light source(s); it is in full light if it sees entirely all the light source(s); otherwise, it is in the penumbra. While the boundary between the penumbra and the full light is reasonably well-understood (see Section 3), less is known about the boundary of the umbra. Nevertheless, there is an extensive literature concerning the explicit computation of these shadow boundaries; see, for example, $[5,7,8,9,11,14$, $16,17]$.

In this paper we prove various bounds, summarized in Table 1, on the complexity of the umbra cast by a segment or polygonal light source on a plane in the presence of polygon or polytope (i.e. convex polyhedral) obstacles. In particular, we show that a single segment light source may cast, in the presence of two triangles, four connected components of umbra. We prove that the umbra defined by one segment light source and two fat convex obstacles of total complexity $n$ can have $\Omega(n)$ connected components. We also prove an $\Omega\left(n k^{2}+k^{4}\right)$ lower bound on the maximum number of connected components of the umbra and a $O\left(n k^{3}\right)$ upper bound on its complexity in a scene consisting of a segment light source and $k$ disjoint polytopes of total complexity $n$. Finally, we prove that the umbra cast on a plane by a polygonal light source and $k$ convex obstacles can have $\Omega\left(n^{2} k^{3}+n k^{5}\right)$ connected components and has complexity $O\left(n^{3} k^{3}\right)$. These are the first bounds on the size of the umbra in terms of both $k$ and $n$.

Our results are surprising in the sense that they show that the umbra cast by a single segment light source may have many connected components. The 
fact that the umbra may have four connected components in the case of two triangle obstacles comes as a total surprise. Our lower bounds of $\Omega\left(n k^{2}+k^{4}\right)$ and $\Omega\left(n^{2} k^{3}+n k^{5}\right)$ connected components, for $k$ polytopes of total complexity $n$, is rather pathological in the sense that most of the obstacles are very long and thin. However, we also present a lower bound example of $\Omega(n)$ connected components in the case of two fat polygons or polytopes of complexity $O(n)$. Concerning our upper bounds of $O\left(n k^{3}\right)$ and $O\left(n^{3} k^{3}\right)$, even though these bounds are not tight, they substantially improve the only previously known bounds for this problem which were the trivial $O\left(n^{4}\right)$ and $O\left(n^{6}\right)$ upper bounds. Finally, it is interesting to point out that even for the simplest case of non-point light sources, obtaining tight bounds on the complexity of the umbra and understanding its structure is a very challenging problem.

The paper is organized as follows. The next section provides notation and definitions. We give in Section 3 almost tight lower and upper bounds on the complexity of the boundary between full light and penumbra cast on a plane by a polygonal light source in the presence of polytope obstacles. We present, in Section 4, upper bounds on the complexity of the umbra, in Section 5, lower bounds on the maximal number of connected components of umbra and conclude in Section 6.

\section{Preliminaries}

Let $s$ be a line segment and $p$ a point. We denote by $\langle s, p\rangle$ the set of line transversals of $s$ through $p$. Similarly, for any triple of segments $s_{1}, s_{2}$ and $s_{3}$, we denote by $\left\langle s_{1}, s_{2}, s_{3}\right\rangle$ its set of line transversals. It is a well-known fact that $\left\langle s_{1}, s_{2}, s_{3}\right\rangle$ consists of lines belonging to the same regulus of a ruled quadric surface (see e.g. [15]). More precisely, the line transversals lie on a hyperboloid of one sheet when the three segments are pairwise skew and not all parallel to the same plane. If the segments are pairwise skew and all parallel to the same plane, then the line transversals lie on a hyperbolic paraboloid. Otherwise, they lie in one or two planes. Hence any set of transversals, whether $\langle s, p\rangle$ or $\left\langle s_{1}, s_{2}, s_{3}\right\rangle$, forms patches of a quadric (possibly degenerating to one or two planes). Moreover, the set of transversals consists of at most three patches, or more formally, at most three connected components in line space [4]. We let $\langle s, p\rangle$ and $\left\langle s_{1}, s_{2}, s_{3}\right\rangle$ denote not just sets of lines but also the surface patches they form.

Let $\mathcal{P}$ be a finite set of disjoint convex polygons or polytopes in $\mathbb{R}^{3}$ with $\mathcal{L} \subset \mathcal{P}$ identified as light sources. A surface $\sigma=\langle e, v\rangle$ is called an EV-surface if there exist two distinct objects $P, Q \in \mathcal{P}$ so that $e$ is an edge of $P, v$ a vertex of $Q$ and $\sigma$ intersects a light source. A surface $\sigma=\left\langle e_{1}, e_{2}, e_{3}\right\rangle$ is called an EEEsurface if there exist three distinct objects $P, Q, R \in \mathcal{P}$ so that $e_{1}, e_{2}$ and $e_{3}$ are respectively edges of $P, Q$ and $R$ and $\sigma$ intersects a light source.

Any plane $\Pi$ intersects an EV-surface or an EEE-surface in a set of arcs of a conic (each possibly empty or possibly a line segment). Hence the intersection between $\Pi$ and all the EV and EEE surfaces defines an arrangement of arcs of 
conics on $\Pi$.

Here we are interested in the arcs of conics that correspond to shadow boundaries. In particular, we are interested in arcs resulting from the intersection between $\Pi$ and maximal free line segments ${ }^{1}$ that intersect a light source and are supported by a line which is on an EV or EEE surface. The intersection of these free line segments with $\Pi$ defines an arrangement of arcs of conics on $\Pi$ which we call the shadow arrangement on the shadow plane $\Pi$.

A point $p$ is in the umbra if for any point $q$ on a light source, the segment $p q$ intersects an object from $\mathcal{P} \backslash \mathcal{L}$. Similarly, $p$ is in full light if for any point $q$ on a light source, the segment $p q$ does not intersect any object from $\mathcal{P} \backslash \mathcal{L}$. Otherwise, $p$ is in the penumbra.

We will make extensive use of the fact that the boundary of the umbra and penumbra consists of arcs of the shadow arrangement (see, for example, [11]). Notice that not all arcs of the shadow arrangement are on the umbra or penumbra boundaries; some arcs correspond to other lighting discontinuities.

Throughout this paper, we consider the regions of umbra and penumbra on a plane cast by a segment light source or polygonal light source(s) in the presence of convex polygons or polytopes.

\section{The penumbra boundary}

We recall here some straightforward and well-known properties of the penumbra and give bounds on the complexity of the common boundary of the penumbra and the full light. In this section we refer to the union of the umbra and penumbra as the shadow region.

Property 1. The shadow region cast by a light source on a plane in the presence of obstacles is the union of all the shadow regions cast by each obstacle.

PROPERTY 2. The shadow region cast on a plane $\Pi$ by a polygonal light source $S$ in the presence of one polytope $P$ is the intersection of half-planes in $\Pi$, each of which is defined as the intersection of $\Pi$ with a half-space that contains $P$ but not $S$, is bounded by a plane tangent to both of them, and contains an edge of one of them.

Note that these two properties imply that the boundary of the shadow region is only composed of line segments induced by EV-surfaces.

Theorem 1 The complexity of the shadow region cast on a plane $\Pi$ by a convex polygonal light source of complexity $m$ in the presence of $k$ convex polyhedra of total complexity $n$ is, in the worst case, in $\Omega\left(n \alpha(k)+k m+k^{2}\right)$ and $O\left(n \alpha(k)+k m \alpha(k)+k^{2}\right)$, where $\alpha(k)$ denotes the pseudo-inverse of the Ackermann function.

\footnotetext{
${ }^{1} \mathrm{~A}$ maximal free line segment is a segment that intersects the interior of no object and whose endpoints lie on some object or at infinity.
} 


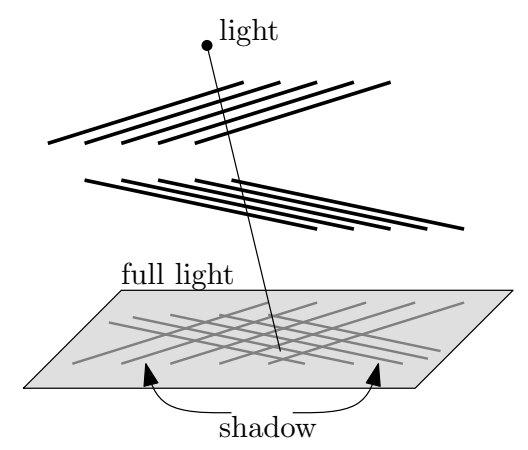

Figure 1: $\Omega\left(k^{2}\right)$ lower bound.

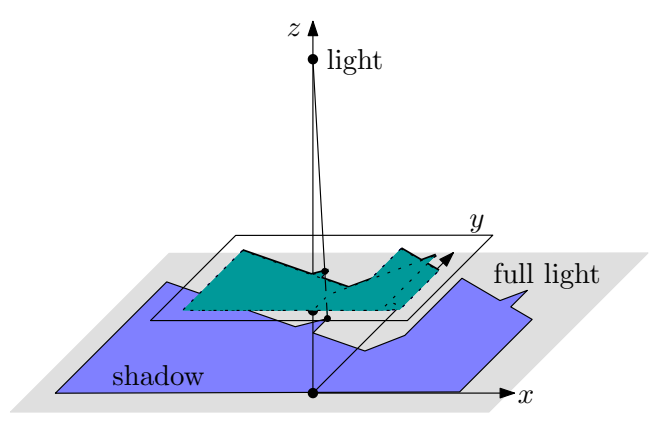

Figure 2: $\Omega(k \alpha(k))$ lower bound.

Proof: By Property 2, the shadow cast on a plane $\Pi$ by a polygonal light source in the presence of one polytope is a convex polygon. Furthermore, if the light source has $m$ edges and the polyhedron has $n_{i}$ edges, the shadow region in $\Pi$ has $O\left(n_{i}+m\right)$ edges. By Property 1 , the shadow region in the presence of $k$ polytopes of total complexity $n$ is thus the union of $k$ convex polygons of total complexity $O(n+k m)$, which has complexity $O\left((n+k m) \alpha(k)+k^{2}\right)[1]$.

For the proof of the stated lower bound consider the following collection of examples. In all constructions the shadow plane $\Pi$ is the plane $z=0$.

$\Omega\left(k^{2}\right)$ example. Refer to Figure 1. We consider a point light source at a height $z$ (large enough) and a grid consisting of $k$ thin horizontal and parallel rectangles at height $z=1$ together with $k$ other thin horizontal and parallel rectangles at height $z=2$. They form a grid of shadow on plane $\Pi$ which has size $O\left(k^{2}\right)$.

$\Omega(k \alpha(k))$ example. Refer to Figure 2. Again, the light source is a point with large positive $z$-coordinate. We consider a set of $k$ line segments in plane $z=1$ (with positive $y$ coordinates) having, in that plane, an upper envelope of size $\Omega(k \alpha(k))[19]$. We transform each line segment into a trapezoid linking it to its projection on the $y=0$ line (in plane $z=1$ ). We get a set of trapezoids whose 
shadow, in plane $z=0$, for a point light source at large enough $z$ is basically the upper envelope of the segments. Note that the trapezoids can easily be made disjoint by placing them in different horizontal planes very close to plane $z=1$.

$\Omega(n \alpha(k))$ example. Refer to Figure 3. First modify the above $\Omega(k \alpha(k))$ example such that the left "vertical" side of each trapezoid has slope $\gamma$ and the right "vertical" side has slope $-\gamma$, for some $\gamma$ large enough. Now, by some suitable scaling, we make all slopes of the vertical walls strictly smaller than $\frac{k \pi}{n}$. Assemble $\frac{n}{k}$ copies of the previous construction into a large regular $\frac{n}{k}$-gon where each side is, in fact, a $k \alpha(k)$ upper envelope. Finally, this construction can be seen as $k$ convex $3 \frac{n}{k}$-gons by connecting all of the $\frac{n}{k}$ copies of the same trapezoid by extending their walls.

This set of $k$ convex $3 \frac{n}{k}$-gons, embedded in different horizontal planes close to the $z=1$ plane, engender, in the presence of a point light source at large enough $z$, shadows of complexity $n \alpha(k)$.

$\Omega(m k)$ example. Refer to Figure 4 . We use a horizontal $m$-gon as light source and a thin rectangle as obstacle. Then the shadow has $\Omega(m)$ size. Using multiple copies of the obstacle such that the different shadows are disjoint easily gives an $\Omega(m k)$ example.

There is still a small gap between the $\Omega\left(k^{2}+m k+n \alpha(k)\right)$ lower bound and the $O\left(k^{2}+m k \alpha(k)+n \alpha(k)\right)$ upper bound. In fact we conjecture that the lower bound is tight; the shadow of the different obstacles have some similarity with homothetic projections of the light, and the union of $k$ convex homothetic $m$ gons is $\Theta(m k)$ since two convex homothetic polygons intersect in at most two points [12].

\section{Upper bounds}

In this section we prove the following two upper bounds on the complexity of the umbra cast by a segment light source or polygonal light source(s) on a plane.

Theorem 2 The complexity of the umbra cast on a plane by one segment light source in the presence of $k$ disjoint polytopes of total complexity $n$ is $O\left(n k^{3}\right)$.

Theorem 3 The complexity of the umbra cast on a plane by a set of $k$ disjoint polytopes of total complexity $n$, some of which are light sources, is $O\left(n^{3} k^{3}\right)$.

\subsection{The umbra cast by a segment light source}

We will actually prove an upper bound on the complexity of the shadow arrangement which yields the same bound for the complexity of the umbra. Notice that, in the case of a single segment light source, the EEE-surfaces $\sigma=\left\langle e_{1}, e_{2}, e_{3}\right\rangle$ and EV-surfaces $\sigma=\langle e, v\rangle$ that contribute to the shadow arrangement are such that either one of $e, e_{1}, e_{2}$ or $e_{3}$ is the segment light source and $v$ is one of its endpoints. 


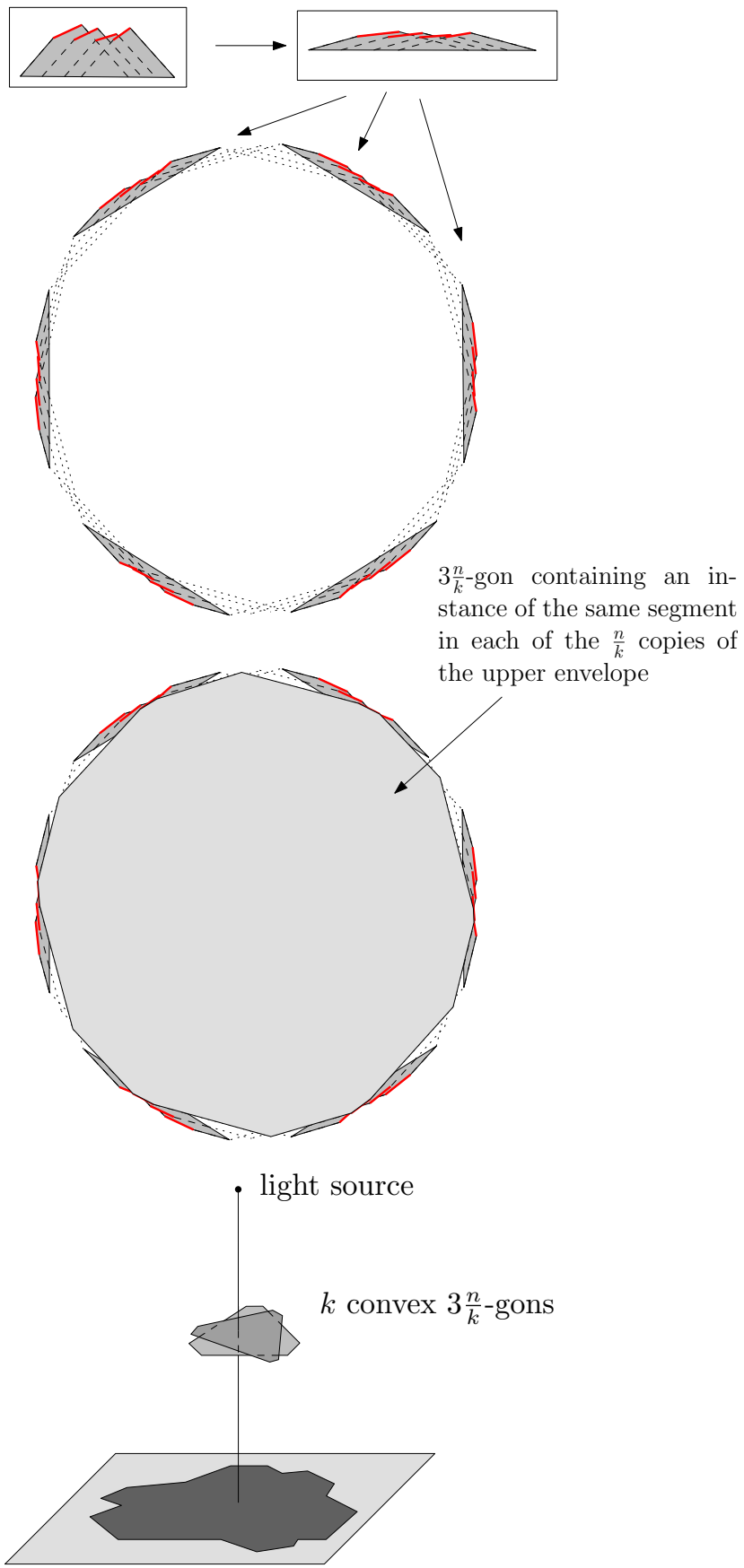

Figure 3: $\Omega(n \alpha(k))$ lower bound. 


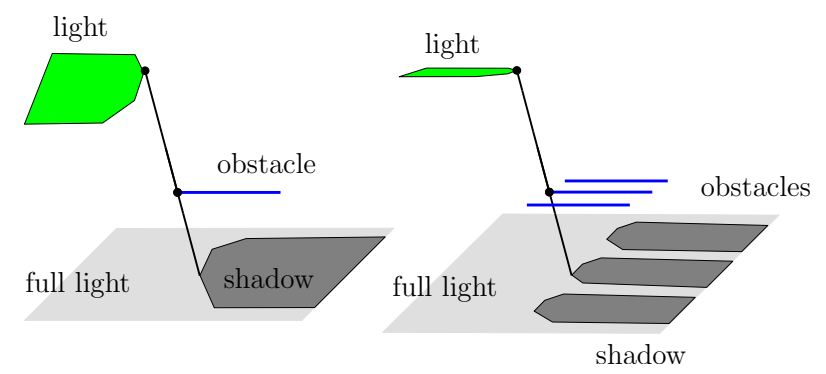

Figure 4: $\Omega(m k)$ lower bound.

We prove Theorem 2 by considering a plane rotating about the line supporting the segment light source. First, if the segment light source, $s$, is not parallel to the shadow plane $\Pi$, we apply a projective transformation to the scene, sending to infinity the point of intersection between the line containing $s$ and plane $\Pi$; this does not change the complexity of the shadow arrangement. We thus assume in the rest of this section that the segment light source is parallel to $\Pi$. The sweep plane, denoted $\pi$, intersects the shadow plane in a line; we will say that, throughout the sweep, this line moves from left to right.

We start with two preliminary lemmas concerning the intersections that one of these sweep planes can have with the shadow arrangement.

Let $\alpha$ be the conic that is the intersection of the shadow plane $\Pi$ and the EEE-surface $\sigma=\left\langle s, s_{1}, s_{2}\right\rangle$ where $s$ is the segment light source, $s_{1}$ and $s_{2}$ are two other segments, and let $\pi$ be a plane containing the light source $s$ and intersecting $\Pi$.

Lemma 4 If $s, s_{1}$, and $s_{2}$ are pairwise skew then $\pi$ intersects $\alpha$ in at most a single point.

Proof: First, since $s$ is parallel to $\Pi$, any transversal to $s$ and to a point in $\alpha \cap \pi$ lies in plane $\pi$. If the intersection between $\pi$ and $s_{1}$ or $s_{2}$ is empty, there exists no line tangent to the three segments in $\pi$ and thus $\pi$ does not cross $\alpha$. Otherwise, both segments intersect $\pi$ in two points $p_{1}$ and $p_{2}$. The line defined by $p_{1}$ and $p_{2}$ is the only line that is possibly a transversal to the three segments in $\pi$ (it may not intersect the segment $s$ ). Hence there exists at most one transversal in $\pi$ which defines exactly one point on $\alpha$.

Notice that the preceding lemma implies that the conic arcs are monotonic in the direction of the sweep (orthogonal to $s$ ).

For the rest of the proof we consider an arrangement $A$ of arcs of conics which contains the shadow arrangement. We will establish an upper bound of $O\left(n k^{3}\right)$ on the complexity of $A$ which will yield the bound of Theorem 2 . The arrangement $A$ consists of the intersection of $\Pi$ with (i) those lines that are transversal to the light source $s$, and the edges $s_{1}$ and $s_{2}$ of two other polytopes and that do not intersect the interior of these polytopes (the connected 
components of these lines form patches of EEE-surfaces) and (ii) those lines that are transversal to a vertex and an edge of two polytopes, one of which is the segment light source, and that do not intersect the interior of these polytopes (the connected components of these lines form patches of EV-surfaces).

We now count the number of crossings between an instance ofthe sweep plane $\pi$ and the $\operatorname{arcs}$ in $A$.

Lemma 5 The plane $\pi$ properly intersects at most $O\left(k^{2}\right)$ arcs of $A$.

Proof: The arcs of $A$ are defined as the intersection with $\Pi$ of lines $\ell$ which are (i) transversal to the segment light source $s$ and tangent to two polytopes, (ii) transversal to an endpoint of $s$ and tangent to another polytope, or (iii) transversal to $s$ and to a polytope vertex.

An instance $\pi$ of the sweep plane never properly intersects an arc of type (iii) (since such an arc is either included in $\pi$ or does not intersect it). Now, if $\pi$ intersects an arc of one of the other two types, then $\pi$ contains the corresponding line $\ell$, which is tangent to two polygons of $\mathcal{P} \cap \pi$. Since $\mathcal{P} \cap \pi$ consists of at most $k$ disjoint convex polygons, there are $O\left(k^{2}\right)$ lines in $\pi$ that are tangent to two polygons of $\mathcal{P} \cap \pi$, hence the result.

Proof:[of Theorem 2] We consider an orthogonal frame in plane $\Pi$ whose vertical axis is parallel to the segment light source $s$; the other axis is called horizontal.

We first show that the number of proper intersection points between arcs of $A$ is $O\left(k^{2}\right)$ times the number of arcs. It follows from Lemma 4 (along with a simple argument in the case that the three segments are not pairwise skew) that any arc of $A$ is either horizontally monotone or is a vertical line segment. Consider an arc $\alpha_{0}$ and its rightmost endpoint $p$ (anyone if $\alpha_{0}$ is vertical). We charge to $\alpha_{0}$ all points of intersection involving $\alpha_{0}$ and all arcs whose rightmost endpoints are strictly to the right of $p$. Any arc properly intersects $\alpha_{0}$ in at most $O(1)$ points so the number of intersection points charged to $\alpha_{0}$ is bounded by the number of arcs properly intersected by the sweep plane containing $p$. By Lemma 5 , there are at most $O\left(k^{2}\right)$ such arcs. Thus, each arc is charged at most $O\left(k^{2}\right)$ times.

We now bound the number of arcs (and thus the number of arc endpoints) generating $A$. Each arc corresponds either to a patch of an EV or EEE surface. Consider first the EV-surfaces. Since either the edge or the vertex is on the light source, there are at most $O(n)$ such surfaces.

Now consider the arcs generated by EEE-surfaces. Let $n_{i}$ be the number of vertices of polytope $P_{i}, 1 \leq i \leq k$. The number of EEE-surfaces involving the light source and edges from polytopes $P_{i}$ and $P_{j}$ is $O\left(n_{i}+n_{j}\right)$ [3, Corollary 2.6] (or [2, Corollary 9]). Then, $\sum_{1 \leq i<j \leq k} O\left(n_{i}+n_{j}\right)=O(n k)$.

There are at most $O(n k)$ arcs generating $A$. Since each arc is charged with at most $O\left(k^{2}\right)$ intersection points, there are at most $O\left(n k^{3}\right)$ intersection points. The total complexity of the shadow arrangement, and thus of the umbra, is then $O\left(n k^{3}\right)$. 


\subsection{The umbra cast by polygonal light sources}

To prove Theorem 3 we consider an arrangement $B$ of arcs of conics that, as in the previous section, contains the shadow arrangement. This arrangement $B$ consists of the intersections of $\Pi$ with (i) the lines that are transversal to a vertex and an edge of two polytopes and that do not intersect the interior of these polytopes (the connected components of these lines form patches of EVsurfaces) (ii) the lines that are transversal to edges of three polytopes and that do not intersect the interior of these polytopes (the connected components of these lines form patches of EEE-surfaces). Notice that $B$ may contain arcs generated by surfaces that do not intersect the light source or possibly by surfaces that intersect the interior of other polytopes in the scene. We will establish a $O\left(n^{3} k^{3}\right)$ upper bound on the complexity of $B$ which yields the same bound for the complexity of the umbra.

Lemma 6 Any line $L$ in $\Pi$ properly intersects at most $O\left(n k^{2}\right)$ arcs of $B$.

Proof: An intersection point between $L$ and $B$ corresponds to a line transversal which belongs to an EV or EEE surface. Consider first EV-surfaces. The line transversal lies in a plane which contains $L$ and a vertex, say $v$, of one of the polytopes. There exist $O(n)$ such planes and in each of them there are at most $O(k)$ lines through $v$ that are tangent to a polytope (since we only consider proper intersections between $L$ and the arcs of $B$ ). Thus there are at most $O(n k)$ points on $L \cap A$ which correspond to lines in EV-surfaces.

Now we consider EEE-surfaces. Let $n_{i}$ be the number of vertices of polytope $P_{i}$, for $1 \leq i \leq k$. The number of EEE-surfaces generated by three edges of polytopes $P_{i}, P_{j}$ and $P_{l}$, not intersecting the interior of $P_{i}, P_{j}$ and $P_{l}$, and that intersect $L$ is $O\left(n_{i}+n_{j}+n_{l}\right)$ [2, Main Lemma]. Since $\sum_{1<i<j<l<k} O\left(n_{i}+n_{j}+\right.$ $\left.n_{l}\right)=O\left(n k^{2}\right)$, there are at most $O\left(n k+n k^{2}\right)=O\left(n k^{2}\right)$ arcs of $B$ which intersect the line $L$ on $\Pi$.

Proof:[of Theorem 3] Here, we introduce an arbitrary coordinate frame $O x y$ in the plane $\Pi$. We call $O x$ the horizontal axis and $O y$ the vertical axis.

As in the proof of Theorem 2, we first show that the number of intersection points between arcs of $B$ is $O\left(n k^{2}\right)$ times the number of conic arcs. We first break all conic arcs into maximal horizontally monotone pieces. This increases the number of arcs only by a constant factor. Consider a conic arc $\alpha_{0}$ and its rightmost endpoint $p$ along $O x$ (any endpoint if the arc is vertical). We charge to $\alpha_{0}$ all points of intersection between $\alpha_{0}$ and all conic arcs whose rightmost endpoints are strictly to the right of $p$. Any arc properly intersects $\alpha_{0}$ in at most $O(1)$ points so the number of intersection points charged to $\alpha_{0}$ is bounded by the number of arcs that are properly intersected by a vertical line in $\Pi$ and containing $p$. By Lemma 6 , there are at most $O\left(n k^{2}\right)$ such arcs. Thus, each arc is charged at most $O\left(n k^{2}\right)$ times.

We now bound the number of arcs (and thus the number of arc endpoints) generating $B$. Let $n_{i}$ be the number of vertices of polytope $P_{i}, 1 \leq i \leq k$ and $e$ an edge. The number of EEE-surfaces pertinent to $B$ and involving $e$ and edges 


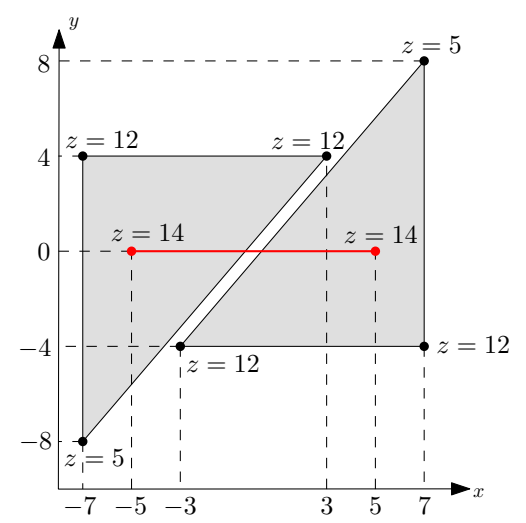

Figure 5: Two triangles and a segment light source (viewed from above) that cast 4 connected components of umbra on the plane $\mathbf{z}=\mathbf{0}$.

from polytopes $P_{i}$ and $P_{j}$ is $O\left(n_{i}+n_{j}\right)$ [2, Corollary 9]. Thus, for each edge $e$, there are, at most, $\sum_{1 \leq i<j \leq k} O\left(n_{i}+n_{j}\right)=O(n k)$ EEE-surfaces having $e$ as a generating segment. Furthermore, the number of EV-surfaces involving edge $e$ or one of its vertices is $O(n)$. Since there exist $n$ edges, the total number of arcs in $B$ is therefore $O\left(n^{2} k\right)$.

In conclusion, there are at most $O\left(n^{2} k\right)$ arcs generating $B$, each of them charged with at most $O\left(n k^{2}\right)$ intersection points, hence there are at most $O\left(n^{3} k^{3}\right)$ intersection points. The total complexity of the $B$ and, thus of the umbra, is $O\left(n^{3} k^{3}\right)$.

\section{Lower bounds}

In this section we present several lower bounds on the complexity of the umbra.

\subsection{The umbra cast by a segment light source}

Here we concentrate on the umbra cast by a segment light source in the presence of various configurations of obstacles.

Theorem 7 A segment light source and two triangles may cast, on a plane, four connected components of umbra.

\section{Proof:}

Consider the following scene consisting of a segment light source, $s$, two triangles, $T_{1}$ and $T_{2}$, and a shadow plane, $\Pi$, the horizontal plane of equation $z=0$; see Figure 5 and Figure 6.

Figure 7 shows a superset of the shadow arrangement generated by this configuration (the arrangement $A$ defined in Section 4). Although it can be 


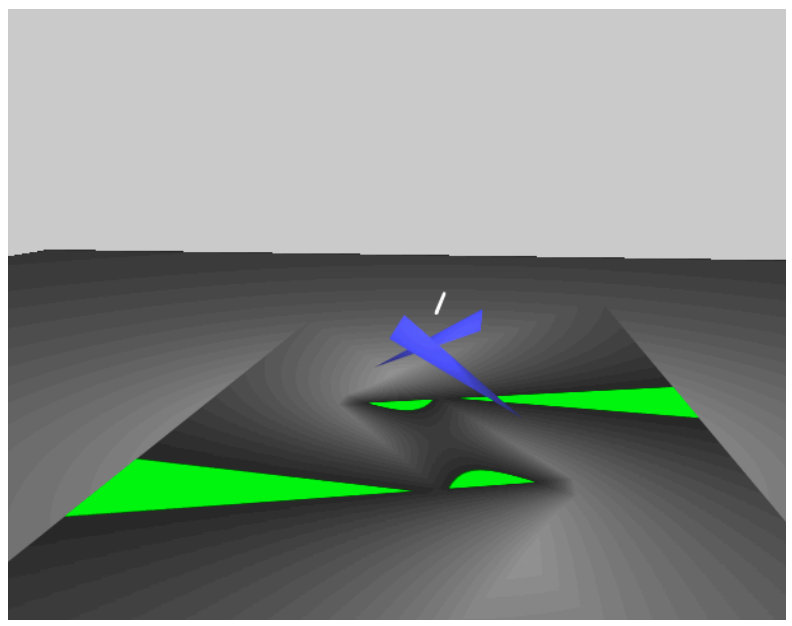

Figure 6: The scene rendered with the ray tracer OpenRT (the umbra is in light grey); courtesy of Andreas Dietrich.

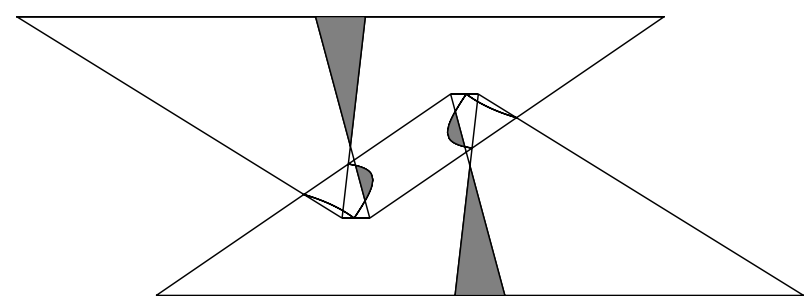

Figure 7: Superset of the shadow arrangement on plane $\Pi$. The four shaded regions are the regions of umbra.

shown that the four shaded regions in the figure are exactly the umbra, we will simply argue here that there are at least four connected components. We do this by illustrating four segments in the umbra and then arguing that they are each in different connected components.

The idea is to consider a series of planes rotating about the segment light source and the intersections of those planes with the two triangles and the shadow plane; Figure 8 shows such a sequence. We then examine the umbra in those planes by considering the relevant bitangents.

Let $P_{+}$be one such plane (containing $s$ ) and going through the point $(0,7,0)$ and $L_{+}$the intersection of $P_{+}$and $\Pi$. Figure $8(\mathrm{~b})$ shows the segment $s$, the intersections between $P_{+}$and the two triangles $T_{1}$ and $T_{2}, L_{+}$and four bitangents that together define the umbra on $L_{+}$. Consider the two segments $R_{1}^{+}$ and $R_{2}^{+}$as shown in Figure 8(b). It is easy to see, by examining the bitangents, that $R_{1}^{+}$and $R_{2}^{+}$are in the umbra. Hence there are two segments of umbra 


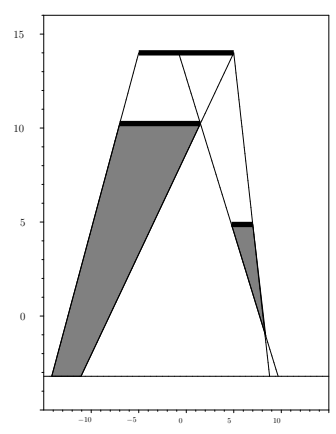

(a)

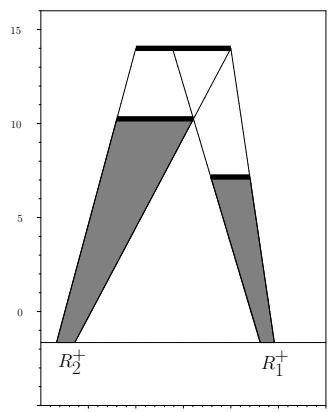

(b)

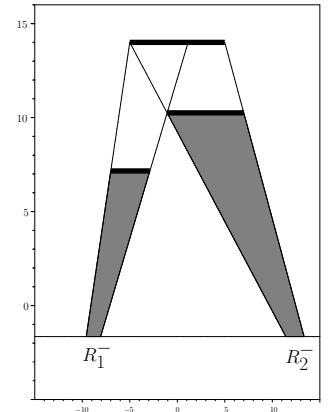

(d)

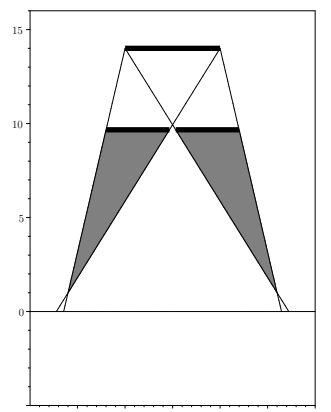

(c)

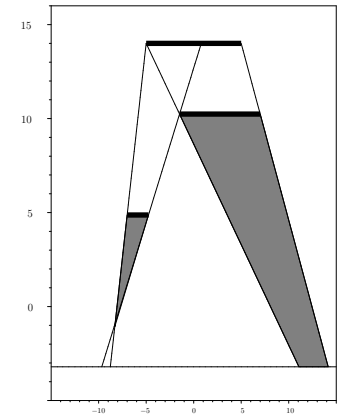

(e)

Figure 8: Views in the sweep plane with bitangents that define the umbra. The number of components of umbra in the intersection of the sweep plane and the plane $\mathbf{z}=\mathbf{0}$ is : (a) one (sweep plane through $(\mathbf{0}, \mathbf{1 0}, \mathbf{0})$ ), (b) two (sweep plane $\mathbf{P}_{+}$through $(\mathbf{0}, \mathbf{7}, \mathbf{0})$ ), (c) zero (sweep plane $\mathbf{y}=\mathbf{0}$ ), (d) two (sweep plane $\mathbf{P}_{-}$ through $(\mathbf{0},-\mathbf{7}, \mathbf{0})),(\mathrm{e})$ one (sweep plane through $(\mathbf{0},-\mathbf{1 0}, \mathbf{0})$ ). 


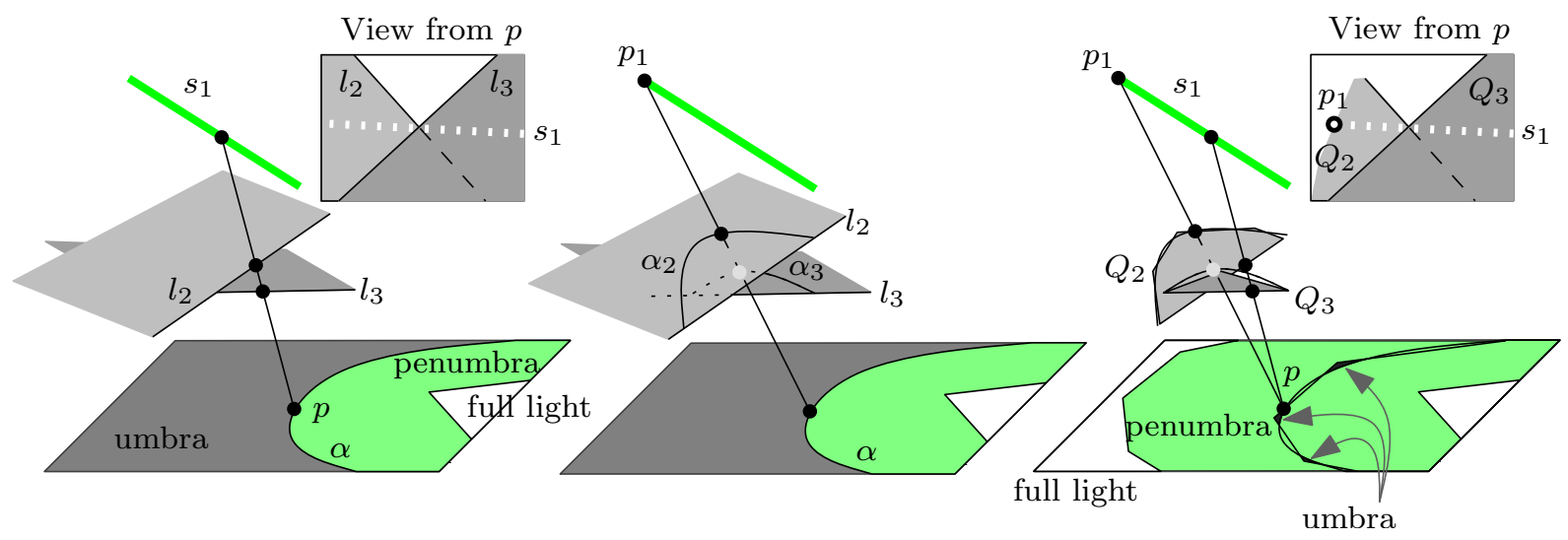

Figure 9: $\Omega(n)$ lower bound.

on the line $L_{+}$. We obtain two other segments, $R_{1}^{-}$and $R_{2}^{-}$, by taking the symmetric plane $P_{-}$with respect to the $x z$-plane (through point $(0,-7,0)$ and whose intersections with the scene is shown on Figure 8(d)).

Now, we show that the four segments $R_{1}^{+}, R_{1}^{-}, R_{2}^{+}$and $R_{2}^{-}$lie in different connected components of umbra. In order to prove this result, we exhibit two lines on $\Pi$ which contain no point in the umbra and separate the four segments as shown in Figure 10.

First consider the plane $y=0$ containing the light segment $s$ and orthogonal to the shadow plane $\Pi$. This plane intersects $\Pi$ in a line, $\delta_{1}$, as shown in Figure 10, and separates $R_{1}^{+}$and $R_{2}^{+}$from $R_{1}^{-}$and $R_{2}^{-}$since $P_{+}$and $P_{-}$are symmetric with the plane $y=0$. To show that $\delta_{1}$ contains no point of the umbra, consider the intersection of the $y=0$ plane with the segment $s$ and the two triangles $T_{1}$ and $T_{2}$; see Figure 8(c). A study of the bitangents reveals that no point of $\delta_{1}$ lies in the umbra.

Now consider the plane orthogonal to $\Pi$, parallel to the two triangle hypotenuses and going through the midpoint of $s$. Let $\delta_{2}$ be the intersection of this plane with $\Pi$; see Figure 10. Elementary computations show that the line $\delta_{2}$ separates $R_{1}^{+}$and $R_{2}^{-}$from $R_{1}^{-}$and $R_{2}^{+}$. There can be no point of the umbra on $\delta_{2}$ since the plane intersects the light source but not the triangles (see Figure 5). This completes the proof.

Note that the line supporting $s$ and the lines supporting the triangle hypotenuses are pairwise skew and not all parallel to a same plane. Thus the corresponding EEE-surface is a hyperboloid of one sheet which intersects $\Pi$ in a hyperbola. We determine the equation of this hyperbolic curve to be $41 y^{2}-52 x y+928=0$. This curve admits two asymptotes which contain no point in the umbra and which separate the connected components of umbra. One of these asymptotes is $\delta_{1}$ and we could have chosen the other to be $\delta_{2}$. 


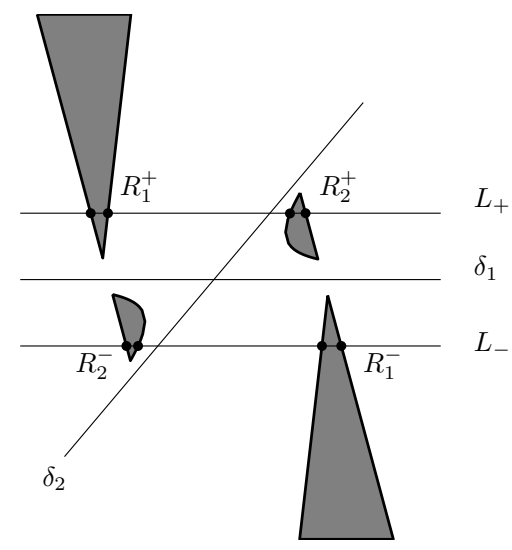

Figure 10: The four connected components of umbra and the four lines used in the proof of Theorem 7 .

Note also that in our example, the light source is parallel to the shadow plane, and there are also many symmetries. None of this is critical; the example can be perturbed and the result still holds.

We know prove a lower bound for fat polytopes, polytopes whose aspect ratios are bounded from below by a positive constant when $n$ goes to infinity.

Theorem 8 The umbra cast on a plane by one segment light source in the presence of two fat disjoint polytopes of total complexity $n$ can have $\Omega(n)$ connected components.

Proof: Our lower-bound example consists of one segment light source $s_{1}$, a polytope $Q_{2}$ of size $O(n)$, and another polytope, $Q_{3}$, of constant size. Refer to Figure 9 .

First we consider three skew lines $l_{1} \supset s_{1}, l_{2}$ and $l_{3}$ and $\sigma=\left\langle s_{1}, l_{2}, l_{3}\right\rangle$ the quadric patch(es) consisting of the lines stabbing $s_{1}, l_{2}$ and $l_{3}$. In the shadow plane $\Pi$, by adding suitable half planes $P_{2}$ and $P_{3}$ as obstacles limited by the lines $l_{2}$ and $l_{3}$, we obtain $\alpha$, a single conic arc of $\sigma \cap \Pi$, bounding the umbra where the umbra is on the concave side of $\alpha$ (Figure 9-left).

We now consider $p_{1}$, one of the endpoints of $s_{1}$, and $\alpha_{2}, \alpha_{3}$, the intersections of planes $P_{2}, P_{3}$ with the cone of apex $p_{1}$ and base $\alpha$ (Figure 9-center).

Next, we reduce the obstacles $P_{2}$ and $P_{3}$ to convex polygons $Q_{2}$ and $Q_{3}$ by limiting them by a polygonal approximation of $\alpha_{2}$ and $\alpha_{3}$ such that $Q_{3}$ remains within (i.e., on the convex side of) $\alpha_{3}$ and $Q_{2}$ intersects $\alpha_{2} n$ times (Figure 9right). The umbra cast by $s_{1}$ on $\Pi$ in the presence of $Q_{2}$ and $Q_{3}$ then consists of $n$ connected components that are the intersection of the concave region outside $\alpha$ and the convex polygon that is the intersection of the cone of apex $p_{1}$ and base $Q_{2}$ with the plane $\Pi$.

Note that the polygons $Q_{2}$ and $Q_{3}$ are fat since $Q_{2}$ consists of a segment and of an approximation of a conic and $Q_{3}$ is of constant size. Finally, polygons 


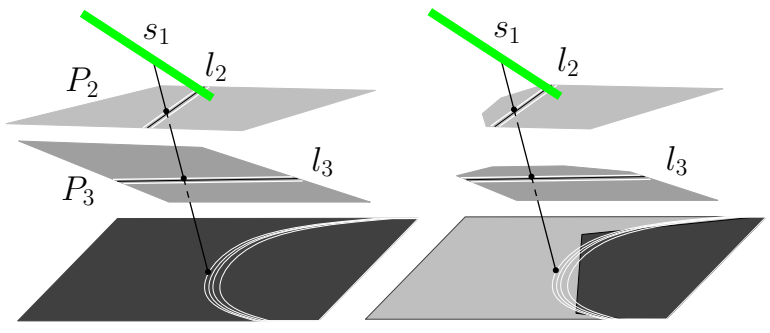

Figure 11: $\Omega\left(n k^{2}\right)$ lower bound.

$Q_{2}$ and $Q_{3}$ can be trivially transformed into fat polytopes without changing the umbra.

Theorem 9 The umbra cast on a plane by one segment light source in the presence of $k$ disjoint polytopes of total complexity $n$ can have $\Omega\left(n k^{2}\right)$ connected components.

Proof: Consider three non-parallel segments $s_{1}, l_{2}$, and $l_{3}$ all parallel to the shadow plane $\Pi$ and planes $P_{2} \supset l_{2}$ and $P_{3} \supset l_{3}$ parallel to $\Pi$, refer to Figure 11. The surface $\left\langle s_{1}, l_{2}, l_{3}\right\rangle$ intersects $\Pi$ in a conic arc $\alpha$.

Now consider the following setup: $s_{1}$ is the light source; $P_{2}$ has $k$ narrow rectangular holes (or slits) parallel and arbitrary close to $l_{2}$; similarly $P_{3}$ has $k$ slits parallel and arbitrary close to $l_{3}$. (A plane with $k$ such slits can be modelled by $O(k)$ rectangles.) $\quad$ Each pair of slits, $s_{2}$ from $P_{2}$ and $s_{3}$ from $P_{3}$, together with the light source $s_{1}$ induce a piece of penumbra in $\Pi$ that is essentially a thickened copy of the conic arc $\alpha$.

We thus get that the umbra covers the whole plane $\Pi$ except for $k^{2}$ curves of penumbra that are all close to $\alpha$ (see Figure 11-left).

Finally, we trim the two planes $P_{2}$ and $P_{3}$, creating an $n$-sided convex polygon on $\Pi$ such that the region outside this polygon is in light or penumbra and each edge intersects all the $k^{2}$ curves. The umbra then consists of $n k^{2}$ regions inside the convex polygon and between the $k^{2}$ conics (see Figure 11-right). Note that the $O(k)$ convex obstacles can each be transformed into a polytope by the addition of a single vertex without changing the umbra.

Theorem 10 The umbra cast on a plane by a segment light source in the presence of $k$ disjoint polytopes can have $\Omega\left(k^{4}\right)$ connected components.

Proof: Refer to Figure 12. As in the previous lower-bound example, we create $k^{2}$ curves of penumbra using parallel thin holes. Making a second set of thin holes in each plane, we create a second family of curves of light and penumbra intersecting the first one. The umbra is now the complement of the union of these two sets of curves and it consists of $\Omega\left(k^{4}\right)$ connected components. 


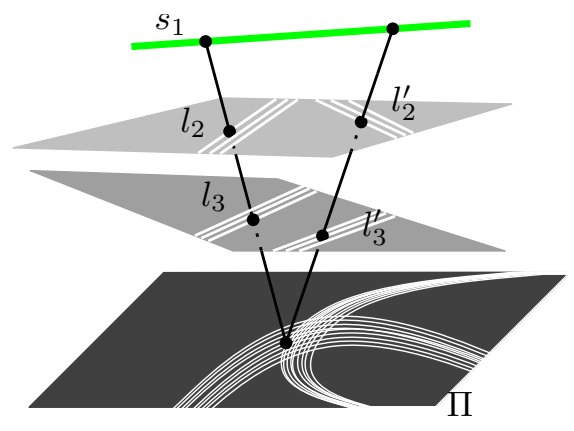

Figure 12: $\Omega\left(k^{4}\right)$ lower bound.

\subsection{The umbra cast by a polygonal light source}

Note that the lower bound of $\Omega\left(n k^{2}+k^{4}\right)$ of Section 5.1 for a segment light source can easily be modified into a lower bound of $\Omega\left(n k^{3}+k^{6}\right)$ in the case of a polygonal light source (by adding a third plane with $O(k)$ slits and a big polygonal light source). We present here a lower bound of $\Omega\left(n^{2} k^{3}+n k^{5}\right)$ on the complexity of the umbra cast by a polygonal light source in the presence of $k$ polygonal obstacles.

Theorem 11 The umbra cast on a plane by one polygonal light source in the presence of $k$ disjoint polytopes of total complexity $n$ can have $\Omega\left(n^{2} k^{3}\right)$ connected components.

Proof: Refer to Figure 13. Let $p$ be a point and $P_{1}$ a small $n$-gon light source very close to $p$. Add a $n$-gon obstacle very close to the light source so that the light source behaves like $n$ point light sources (when viewed from the correct side).

Now consider a plane obstacle with $k$ thin holes parallel to a line $l_{1}$. This creates $n k$ parallel thin lines of light on the shadow plane that can be made arbitrarily close to a line $L$ (by having the $k$ thin holes sufficiently close to each other and the $n$ point light sources sufficiently close to each other). Note that by duplicating this construction (and thus with two polygonal light sources which behave as $2 n$ point light sources) we get an arrangement of $2 n k$ lines of light with $n^{2} k^{2}$ connected components of umbra.

Now consider two lines $l_{2}$ and $l_{3}$ that together with $L$ admit a quadric as line transversals. Cut this quadric by a plane and approximate (a piece $C$ of) the resulting conic by a convex $n$-polyline, $P_{2}$. The set of transversals to the boundary of this polyline with $l_{2}$ and $l_{3}$ defines a curve on the shadow plane that cuts $L$ order $n$ times. We define a light source as the convex hull of $P_{2}$ and put an obstacle very close to it so that the light source behaves as if the polyline $P_{2}$ was the light source (when viewed from the right region). Now, replacing $l_{2}$ and $l_{3}$ by two plane obstacles with order $k$ thin holes close and parallel to 

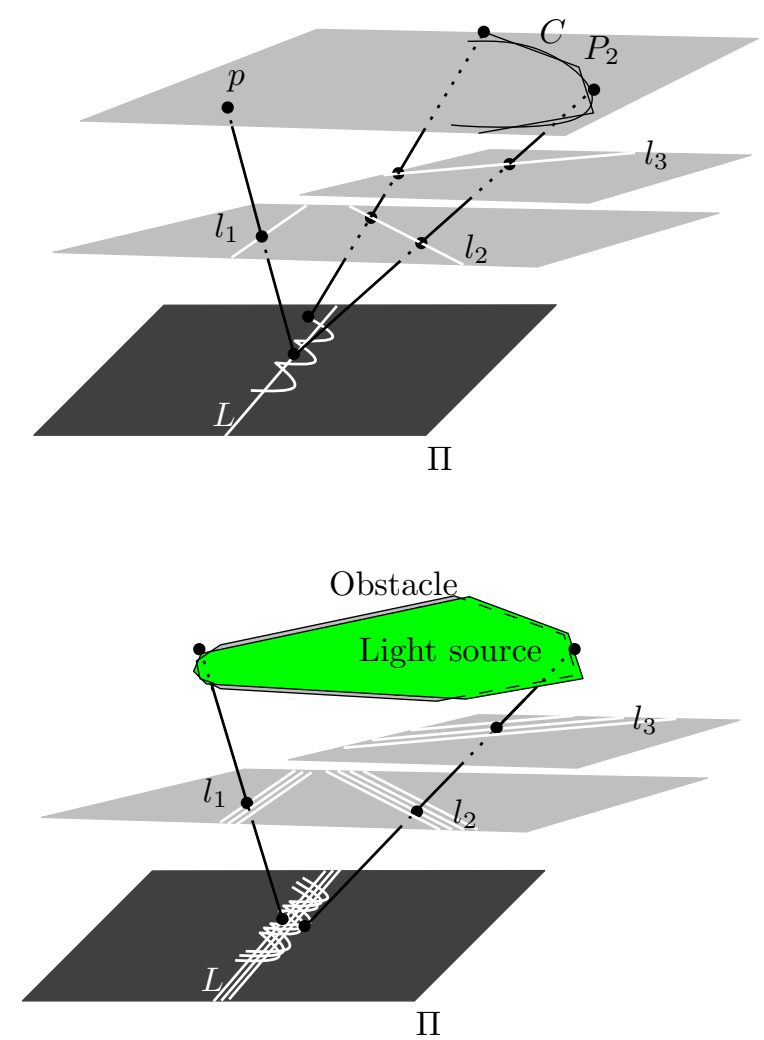

Figure 13: $\Omega\left(n^{2} k^{3}\right)$ lower bound.

$l_{2}$ and $l_{3}$, respectively, we get $k^{2}$ curves of light, each of which intersects order $n$ times each of the $n k$ lines of light close to $L$. This give $\Omega\left(n^{2} k^{3}\right)$ connected component of the umbra.

Note that the two light sources $P_{1}$ and $P_{2}$ can be merged into one by considering $P_{2}$ in the same plane as $P_{1}$, by noticing that there are enough degrees of freedom on $l_{1}$ and $l_{2}$ so that the convex hull of $P_{1}$ and an arc of the conic contains $C$ on its boundary.

Theorem 12 The umbra cast on a plane by one polygonal light source in the presence of $k$ disjonit polytopes of total complexity $n$ can have $\Omega\left(n k^{5}\right)$ connected components.

Proof: Refer to Figure 14. Consider three horizontal pairwise skew lines $l_{1}$, $l_{2}, l_{3}$ that lie above a horizontal plane $\Pi$ and let $C$ be the conic intersection of their common transversal with $\Pi$. Replace each of the $l_{i}$ by a plane obstacle and $k$ thin holes close to $l_{i}$ and place a large (horizontal) light source $S$ above these planes obstacles. 

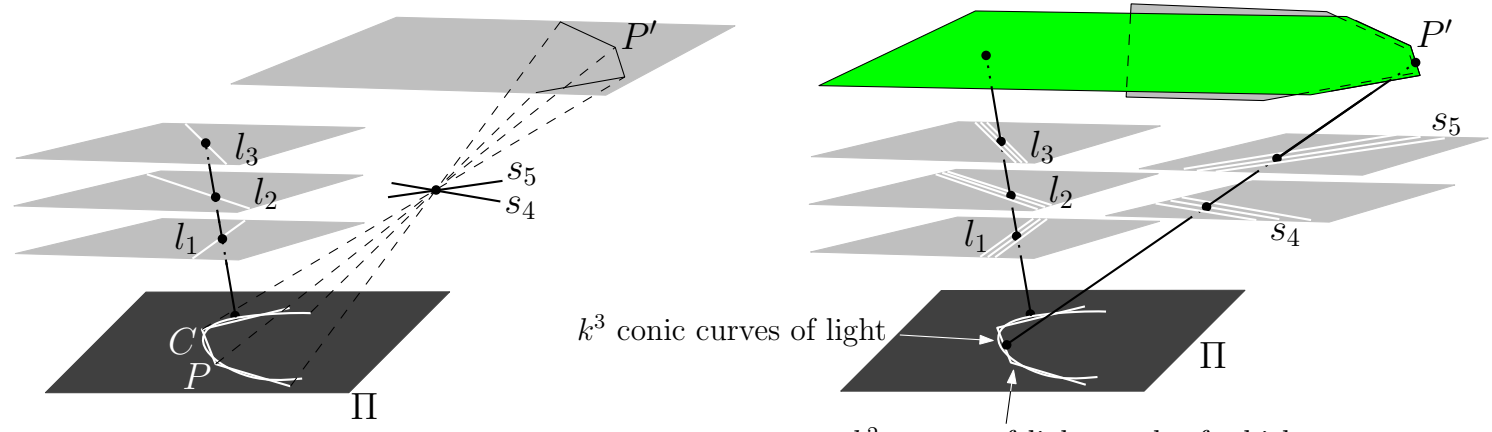

$k^{2}$ curves of light, each of which consists of $\Theta(n)$ conic arcs that each intersects each of the $k^{3}$ conic curves of light

Figure 14: $\Omega\left(n k^{5}\right)$ lower bound.

Consider now a $n$-gon $P$ that intersects $C$ order $n$ times. Let $s_{4}$ and $s_{5}$ be two intersecting horizontal segments. Let $P^{\prime}$ be the symmetric of $P$ with respect of the point of intersection between $s_{4}$ and $s_{5}$. We consider $P^{\prime}$ as a light source and put an obstacle very close to it so that it behaves as a one-dimensional polygonal light source when viewed from $C$. This induces on the shadow plane a polyline of light that intersects $C$ order $n$ times.

Now perturb segments $s_{4}$ and $s_{5}$ so that they do not intersect and replace them by (horizontal) plane obstacles with $k$ thin holes close and parallel to $s_{4}$ and $s_{5}$, respectively. We hence get $k^{2}$ curves of light, each of which consists of order $n$ conic arcs that each intersects $C$; hence each of these $k^{2}$ curves of light intersects $C$ order $n$ times. By chosing the holes near $l_{1}, l_{2}$ and $l_{3}$ sufficiently close to each other, respectively, each of the $k^{2}$ curves of light close to $P$ intersects $O(n)$ times each of the $k^{3}$ curves of light close to $C$. We hence get $n k^{5}$ connected components of umbra.

\section{Conclusion}

The purpose of this paper is to establish the complexity of the boundaries between the umbra, penumbra and fully-lit regions on a plane in a polyhedral scene consisting of $k$ convex objects of total complexity $n$.

The results presented here constitute a first step toward understanding the intrinsic structure and complexity of the umbra in this setting. We have proved that if the light is reduced to one line segment, then the umbra may have $\Omega\left(n k^{2}+k^{4}\right)$ connected components and $O\left(n k^{3}\right)$ complexity. We have also shown that a polygonal light source could generate an umbra with $\Omega\left(n^{2} k^{3}+n k^{5}\right)$ connected components and $O\left(n^{3} k^{3}\right)$ complexity. In both cases these components 
of umbra are delimited by arcs of conics. These results prove that the umbra is intrinsically much more intricate than the boundary between full light and penumbra which is bounded by line segments and has complexity $O(n \alpha(k)+$ $k m \alpha(k)+k^{2}$ ), where $m$ is the complexity of the light source.

Our upper bounds, in fact, apply to the complexity of the arrangement of the curves where the derivative of the light intensity is discontinuous. These arrangements clearly include the boundary of the umbra, but also a lot of curves inside the penumbra that are not relevant to the umbra. Furthermore, our upper bound on the complexity of these arrangements is tight for a segment light source (see the full paper for details). This perhaps explains why our bounds on the complexity of the umbra are not tight. Notice, however, that we do have tight bounds for small $k(k=O(1))$ and for small $n(n=O(k))$.

\section{Acknowledgments}

Many thanks to Jeff Erickson for useful discussions during the early stages of this work.

\section{References}

[1] B. Aronov and M. Sharir. The common exterior of convex polygons in the plane. Computational Geometry: Theory and Applications, 8:139-149, 1997.

[2] H. Brönnimann, O. Devillers, V. Dujmović, H. Everett, M. Glisse, X. Goaoc, S. Lazard, H.-S. Na, and S. Whitesides. On the number of maximal free line segments tangent to arbitrary three-dimensional convex polyhedra. Research Report $\mathrm{n}^{\circ}$ 5671, INRIA, Sept. 2005. 28 pages.

[3] H. Brönnimann, O. Devillers, V. Dujmović, H. Everett, M. Glisse, X. Goaoc, S. Lazard, H.-S. Na, and S. Whitesides. Lines and free line segments tangent to arbitrary three-dimensional convex polyhedra. SIAM Journal on Computing, 2006. To appear.

[4] H. Brönnimann, H. Everett, S. Lazard, F. Sottile, and S. Whitesides. Transversals to line segments in three-dimensional space. Discrete and Computational Geometry, 34(3):381-390, 2005.

[5] G. Drettakis and E. Fiume. A fast shadow algorithm for area light sources using backprojection. In Computer Graphics Proceedings, Annual Conference Series, ACM SIGGRAPH, pages 223-230, New York, 1994. ACM Press.

[6] F. Durand. A multidisciplinary survey of visibility, 2000. ACM SIGGRAPH course notes, Visibility, Problems, Techniques, and Applications. 
[7] F. Durand, G. Dretakkis, and C. Puech. The visibility skeleton : a powerful and efficient multi-purpose global visibility tool. In Computer Graphics Proceedings, Annual Conference Series, ACM SIGGRAPH, pages 89-100, New York, 1997. ACM Press/Addison-Wesley Publishing Co.

[8] F. Durand, G. Dretakkis, and C. Puech. Fast and accurate hierarchical radiosity using global visibility. ACM Transactions on Graphics, 18(2):128170, 1999.

[9] F. Durand, G. Dretakkis, and C. Puech. The 3D visibility complex. ACM Transactions on Graphics, 21(2):176-206, 2002.

[10] J-M. Hasenfratz, M. Lapierre, N. Holzschuch, and F. Sillion. A survey of real-time soft shadows algorithms. In Eurographics, 2003.

[11] P. S. Heckbert. Discontinuity meshing for radiosity. In Proceedings of the Third Eurographics Workshop on Rendering, pages 203-215, May 1992.

[12] K. Kedem, R. Livne, J. Pach, and Micha Sharir. On the union of Jordan regions and collision-free translational motion amidst polygonal obstacles. Discrete and Computational Geometry, 1:59-71, 1986.

[13] P. Mamassian, D. C. Knill, and D. Kersten. The perception of cast shadows. Trends in Cognitive Sciences, 2(8):288-295, 1998.

[14] T. Nishita and E. Nakamae. Half-tone representation of 3D objects illuminated by area sources or polyhedron sources. In IEEE Computer Society's 7th International Computer Software and Applications Conference (COMPSAC '83), pages 237-242, 1983.

[15] G. Salmon. Analytic Geometry of Three Dimensions. Cambridge, 1978.

[16] A. J. Stewart and S. Ghali. Fast computation of shadow boundaries using spatial coherence and backprojections. In Computer Graphics Proceedings, Annual Conference Series, ACM SIGGRAPH, pages 231-238. ACM Press, 1994.

[17] S. J. Teller. Computing the antipenumbra of an area light source. In Computer Graphics Proceedings, Annual Conference Series, ACM SIGGRAPH, pages 139-148. ACM Press, 1992.

[18] L. Wanger. The effect of shadow quality on the perception of spatial relationships in computer generated images. Computer Graphics, 25(2):39-42, 1992.

[19] A. Wiernik and Micha Sharir. Planar realizations of nonlinear DavenportSchinzel sequences by segments. Discrete and Computational Geometry, 3:15-47, 1988.

[20] A. Woo, P. Poulin, and A. Fournier. A survey of shadow algorithms. IEEE Computer Graphics and Applications, 10(6):13-32, 1990. 